\title{
Haptic Models of an Automotive Turn-Signal Switch: Identification and Playback Results
}

\author{
Mark B. Colton ${ }^{*} \quad$ John M. Hollerbach ${ }^{\dagger}$ \\ (*)Department of Mechanical Engineering, Brigham Young University, USA \\ (†) School of Computing, University of Utah, USA \\ E-mail:colton@byu.edu,jmh@cs.utah.edu
}

\begin{abstract}
Creating accurate haptic models of physical devices is critical to improving the realism and immersiveness of virtual environments. This paper addresses the problem of modeling the feel of an automotive turnsignal switch, a prototypical device chosen for its strongly nonlinear dynamic behavior. Parameters of a position- and direction-dependent nonlinear impedance model are estimated from experimental data using a modified version of the exponentiallyweighted least-squares (EWLS) algorithm. The method is then validated through playback experiments in which subjects rate the realism of the resulting models.
\end{abstract}

\section{Introduction}

Typical buttons and switches exhibit a complex feel characterized by dynamics that are often highly nonlinear. These nonlinearities may include friction, backlash, detents, variable stiffness, and other effects that contribute to the characteristic feel of a particular switch. The nonlinear effects are often purposely introduced by the designer to augment the functionality of the button or switch. For instance, detents provide to the user a haptic indicator of the switch state, hardening springs give a sense of the position along the switch's stroke, mechanical stops signal to the user that the end of the stroke has been reached, and friction adds energy dissipation and smoothness to the switch's operation. The nonlinearities specific to a given switch also serve to set its feel apart from the feel other switches, allowing the user to manually discriminate between controls, and manipulate them in complex environments, without relying on visual senses alone.

Switches are present in nearly all real environments, which suggests that adding haptic models of switches to virtual environments has the potential to create more immersive and useful simulations. Virtual prototyping, in which users design and test products virtually, is an example of an application in which haptic switch models would be useful. Consider the specific example of designing an automobile interior. A physical prototype that allows designers to evaluate the interior aesthetics and the placement of controls would necessarily be costly, and design changes would require additional physical prototypes to be built for subsequent evaluation. A virtual prototype, on the other hand, would allow more rapid modification and evaluation of each design iteration. To be truly useful, the virtual prototype would require accurate models of the primary automotive controls, including the steering wheel, gear shift, turn-signal switch, and others.

\subsection{Related work}

Modeling of switches and knobs has been addressed by other researchers within and outside the haptics community. Allotta et al. [1] simulated the feel of a cam-based rotary knob, where the model was derived using first principles. Angerilli et al. [2] recorded the force-position profile of an automotive gearshift lever, and played back the recorded profile on a specialized haptic device. Weir et al. [3] used a human-actuated instrumented probe to record force profiles of pushbutton switches, and plotted the forces against position, velocity, and acceleration. Nagurka and Marklin [4] measured the force characteristics of computer keyboard keys, actuated with constant velocity inputs using an instrumented probe. MacLean [5] obtained static segmented force models of a toggle switch from experimental measurements.

\subsection{Objective}

The objective of the present work is to develop a method to obtain haptic models of buttons and switches from experimental data. Specifically, haptic models of a common automotive turn-signal switch, such as that shown in Fig. 1, have been obtained. The 
basis for this work has been described in [7] and [8], in which a general nonlinear model structure and parameter estimation method were introduced, and a one degree-of-freedom (DOF) instrumented probe was described. Initial experiments showed that the methods are able to accurately model a custom-built nonlinear calibration system [7] and two general pushbutton switches [8].

The underlying aim of this work is to develop methods that are both general (in the sense that they may be applied to a wide variety of physical devices) and accurate (in the sense that they generate models that closely replicate the feel of the devices being modeled). The approach taken in this work is to use experimental force and motion measurements to estimate parameters of a general, dynamic, nonlinear model. The advantage of this approach over simply recording and playing back a switch's force profile is that dynamic effects may be modeled, whereas static recordings do not account for forces that vary with varying operating conditions, such as inertial or damping forces. The method is also automatic and versatile, and does not rely on a priori knowledge of the switch's internal structure. Although these methods have been applied to the specific problem of modeling the feel of a turn-signal switch, it is anticipated that the same model structure, perturbation inputs, and parameter estimation algorithm may be used to model a much wider class of target devices.

\section{Apparatus}

The modeling target, a standard turn-signal switch, is shown in Fig. 1. Details of the internal structure of the switch are shown in Fig. 2. As the user actuates the turn-signal switch via the lever, a spring-loaded follower is caused to follow a stationary cam surface with three detent positions, as shown in the figure. This cam surface is the primary contributor to the distinctive feel and nonlinear dynamics of the turnsignal switch. The three detents correspond to the three switch states: left turn, right turn, and neutral (no

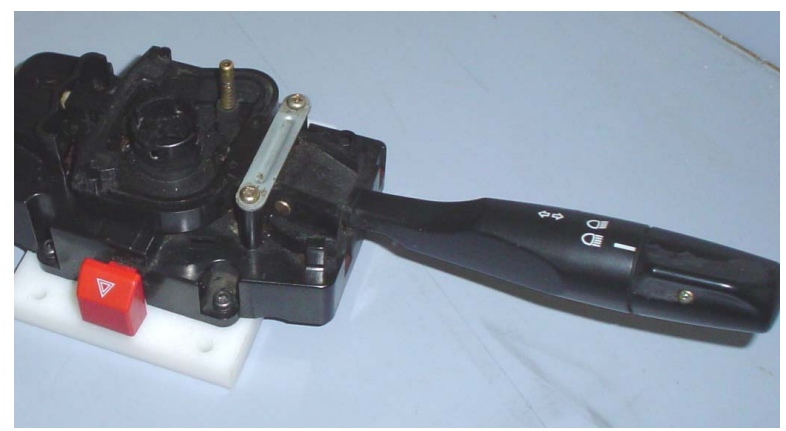

Fig. 1. Turn-signal switch

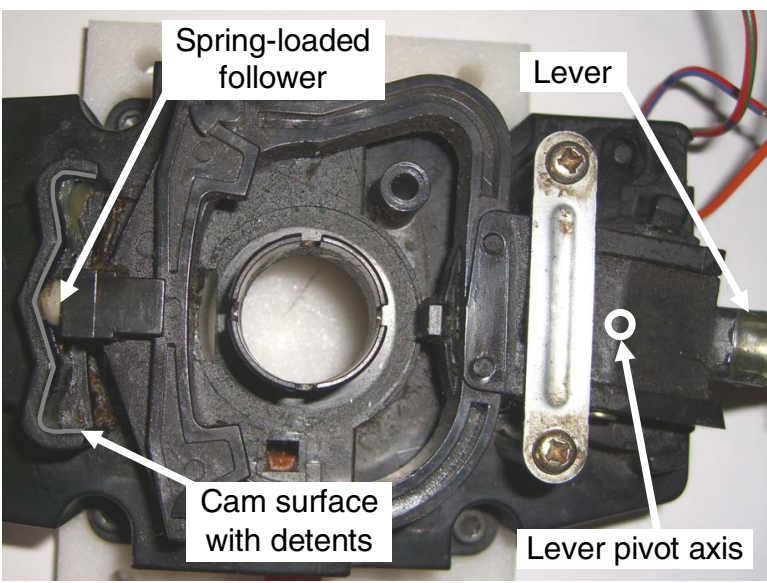

Fig. 2. Internal structure of turn-signal switch

turn). Other sources of nonlinearities include friction and backlash, both of which are discernible when actuating the device. The stroke of the switch is approximately $35 \mathrm{~mm}$, and the maximum static actuation force was measured to be approximately 8.9 $\mathrm{N}$.

A 1-DOF instrumented probe was designed to apply position trajectories to the turn-signal switch and record the resulting force [6]. The probe consists of a direct-drive linear motor for actuating the target device, an incremental encoder for measuring position, and an impedance head for measuring force and acceleration. The experimental setup is shown in Fig. 3 . The flexible coupling shown in the figure maintains axial rigidity to ensure accurate measurements, while providing lateral and angular flexibility to aid in interfacing with the turn-signal switch.

\section{Methods}

\subsection{Model structure}

As mentioned previously, a general, nonlinear, dynamic, impedance-type model structure was developed for modeling the feel of general passive, mechanical devices [6]. The model is repeated here:

$$
F= \begin{cases}m^{+}(x) a+b^{+}(x) v+k^{+}(x) x+F_{o}^{+}(x), & v>0 \\ m^{-}(x) a+b^{-}(x) v+k^{-}(x) x+F_{o}^{-}(x), & v<0\end{cases}
$$




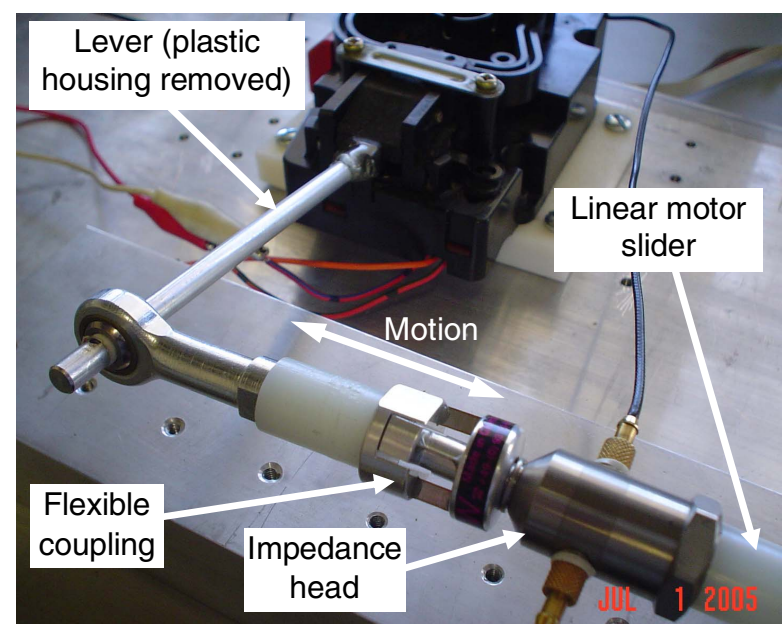

Fig. 3. Experimental setup

In (1), the force $F$ is a nonlinear function of the position $x$, velocity $v$, and acceleration $a$. The model parameters $m$ (mass), $b$ (damping), $k$ (stiffness), and $F_{o}$ (offset force) are allowed to vary with position and direction, with the superscripts indicating the direction of travel for which the model parameters are valid. Equation (1) is, in essence, a position- and directiondependent mass-spring-damper model, derived by linearizing a general nonlinear force function about a varying operating point [6]. The model was selected for its versatility, and has been shown to accurately model the feel of push-button switches [7].

\subsection{Parameter estimation method}

The method for estimating the position- and direction-varying parameters in (1) is based on the exponentially-weighted least-squares (EWLS) method, as described in [6] and [7]. The EWLS algorithm is designed to estimate time-varying parameters of linear models, and is often applied in real-time estimation applications. The challenge in the present work was to modify the EWLS algorithm to make it capable of estimating position- and direction-dependent parameters of the nonlinear model described by (1). This is accomplished by grouping by direction the data obtained by actuating the turn-signal switch using the instrumented probe described in Section 2. The result is two data sets, one containing the data corresponding to motion in the positive direction, and one containing data from measurements in the negative direction. Each data set is then sorted by direction. The EWLS algorithm is applied sequentially to each data set, resulting in a set of estimated model parameters $(m, b$, $k$, and $F_{o}$ ) corresponding to each measured data point in each direction. The result is a table of thousands of parameter estimates, many of which are at repeated $x$ locations due to the periodic nature of the perturbation inputs. These model parameters are then resampled at a spacing $\Delta x$, called the resampling increment.

The nature of the parameter estimates obtained using EWLS is controlled through the selection of a constant parameter $\lambda$, called the forgetting factor, which takes on a value between 0 and 1 . Small values of $\lambda$ cause less weighting of past data points, resulting in greater responsiveness to changing model parameters, but lower immunity to noise. Large values of $\lambda$ cause greater weighting of past data points, resulting in smoother model parameter estimates and greater noise rejection.

\section{Modeling experiments}

The instrumented probe described previously was used to actuate the turn-signal switch according to several predetermined motion trajectories and record the resulting force. The trajectories were designed to: 1) ensure that the probe traverses the entire $x$-range of the turn-signal switch; 2) contain a range of frequencies that adequately excite the dynamics of the target device. For devices with an unknown nonlinear model structure (and, therefore, unknown frequency requirements), the approach taken was to create trajectories that contained a wide range of frequencies, while remaining within the capabilities of the instrumented probe and safety limits of the target device. Candidate trajectories included singlefrequency sinusoids, swept sines, pseudo-random binary sequences (PRBS), and hybrid combinations of sinusoids, swept sines, and PRBS. The results presented here are for a $1-4 \mathrm{~Hz}$ swept sine, of amplitude $4 \mathrm{~mm}$, superimposed on a $0.5 \mathrm{~Hz}, 12 \mathrm{~mm}$ sine.

Using the methods discussed in previous sections, models were generated based on this input for multiple values of the forgetting factor $(\lambda=0.999 \ldots 0.9999)$ and resampling increment $(\Delta x=0.05 \ldots 4 \mathrm{~mm})$. The forces predicted by each model were compared to the measured forces generated using a $1.5 \mathrm{~Hz}$ validation position trajectory. The variance-accounted-for (VAF), which represents the proportion of the variance of the force that is predicted by the model [8], was calculated for each model realization. The results are shown in Fig. 4, which shows the effects of the forgetting factor and resampling increment on the quality of the model, as measured by the agreement of the predicted force and measured force. Among all model realizations, the model based on $\Delta x=0.5 \mathrm{~mm}$ and $\lambda=0.9990$ was found to achieve the highest VAF (0.9241). The estimated model parameters that resulted from this combination of $\lambda$ and $\Delta x$ are shown in Fig. 5. The 
nonlinear nature of the model is apparent in this figure, as demonstrated by the considerable variation in the model parameters across the turn-signal's range and direction of travel. Fig. 6 shows the forces predicted by this model in response to a $1.5 \mathrm{~Hz}$ validation trajectory, compared with the forces measured during the same validation experiment. Based on this plot, it appears that the model satisfactorily describes the feel of the turn-signal switch. Of equal interest is the model's ability to represent the frequency content of the dynamic forces. To evaluate the model's frequency-domain capabilities, the Fast Fourier Transform (FFT) of the measured and predicted forces was calculated, as shown in Fig. 7. The effect of the forgetting factor on the nature of the parameter estimates is shown in Fig. 8. As anticipated, larger values of forgetting factor result in smoother model parameter profiles.

\section{Playback experiments}

An initial set of playback experiments was performed to validate the quality of the models developed using the methods described in this paper. The experiments sought to address the following questions: 1) Does the modified EWLS method yield models that qualitatively feel like the target device that has been modeled? 2) Does the VAF error measure predict which model realizations are preferred by users? 3) Is there a relationship between forgetting factor and model realism, as measured by the user responses? To answer these questions, 10 subjects $(8$ male, 2 female, mean age of 25.6 years) were asked to rate the realism of models that were played back to them on a scale of 1 to 7 , with a rating of 7 corresponding to "very realistic," and 1 corresponding to "not at all realistic." The model realizations were based on forgetting factors of $\lambda=\{0.9991,0.9993$, $0.9995,0.9997,0.9999\}$ and a resampling increment of $\Delta x=1 \mathrm{~mm}$. These five models were played back in

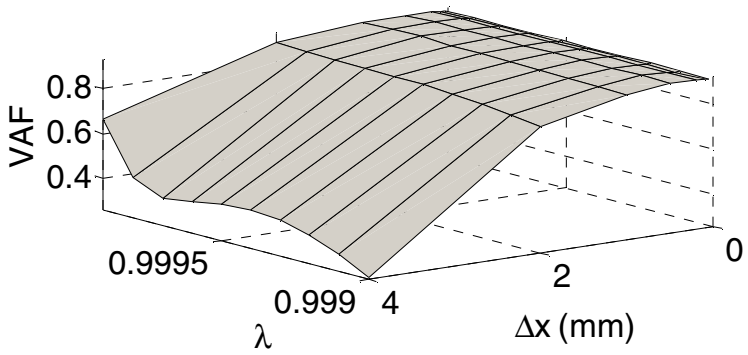

Fig. 4. Variance-accounted-for (VAF) based on measured and predicted forces as a function of forgetting factor and resampling increment
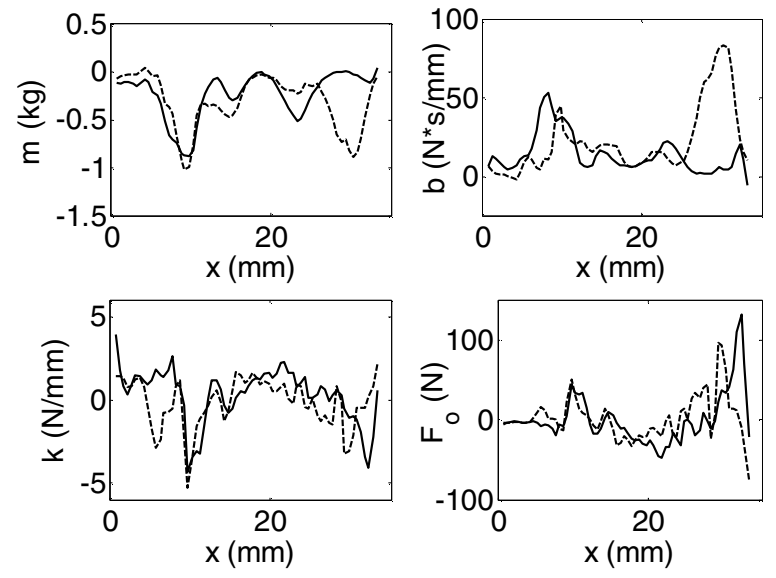

Fig. 5. Estimated nonlinear model parameters. Solid line: positive-direction. Dashed line: negative direction

random order, repeated three times, resulting in 15 trials per subject. After completing all trials, the average rating for each model realization was calculated.

The instrumented probe described previously was used in these experiments as a haptic playback device, with a circular thimble-type grip attached to the end of the linear motor's slider (see Fig. 9). To assist the subjects in determining the realism of each turn-signal model realization, the physical turn-signal switch was situated next to the haptic playback device, and the subjects were asked to actuate the physical switch between each haptic trial. No limit was imposed on the amount of time that subjects could actuate and feel the physical switch or the haptic device. After each trial, the subject reported the realism rating, and a new model realization was displayed as soon as the subject was ready.

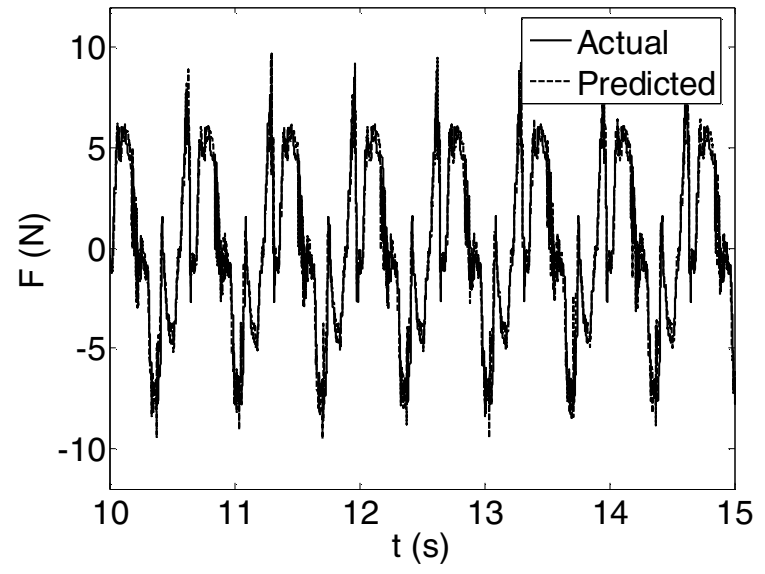

Fig. 6. Measured and predicted forces based on the turn-signal switch model 


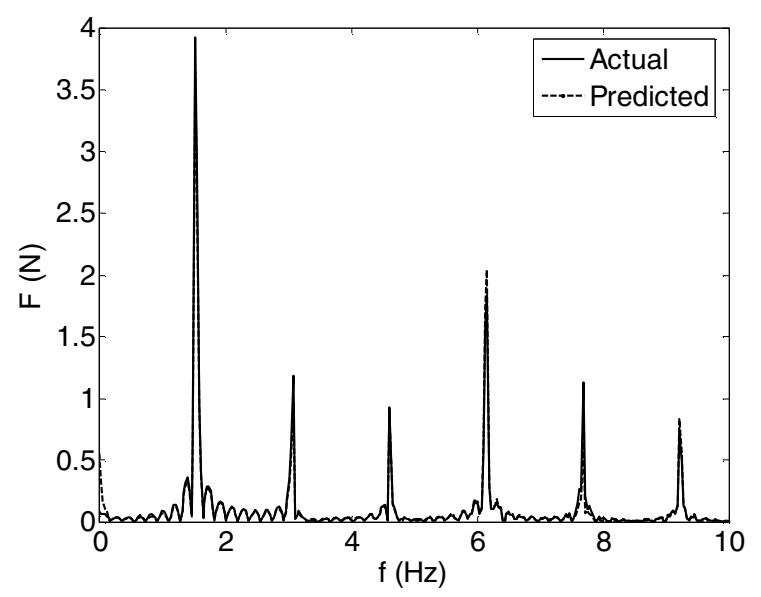

Fig. 7. Measured and predicted FFT of forces based on the turn-signal switch model

The mean model rating for all subjects is shown in Fig. 10, where the error bars represent one standard deviation from the mean. Note that the model based on a forgetting factor of $\lambda=0.9995$ received the highest average rating (4.800) and the lowest standard deviation (0.997). Fig. 11 shows the same results (upper plot) together with the VAF obtained from the identification experiments described in Section 4 (bottom plot). As shown in the plot, the highest user rating and lowest standard deviation occurred for the same model that achieved the highest VAF for this particular value of the resampling increment $(\Delta x=1$ $\mathrm{mm}$ ). This may suggest that the VAF, which is a measure of the goodness of fit of the model, may be an indicator of the ability of the model to replicate the feel
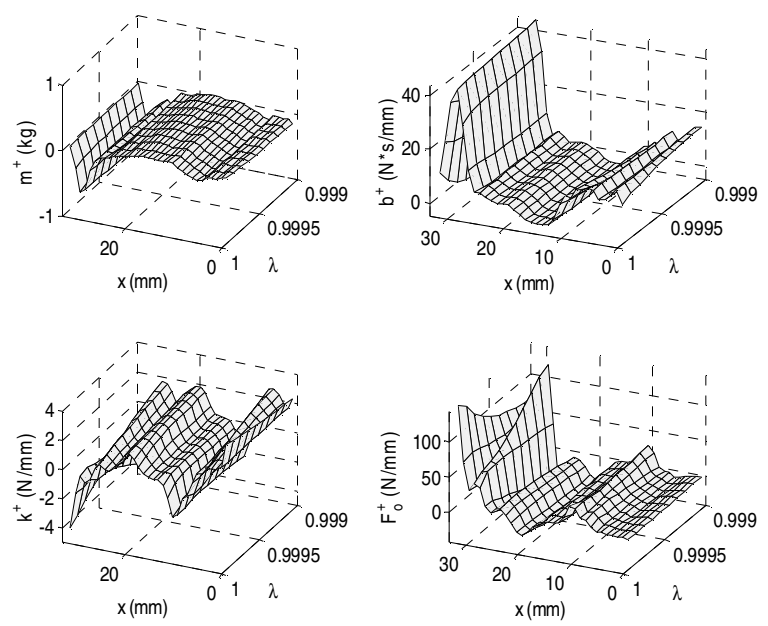

Fig. 8. Effects of forgetting factor on model parameters.

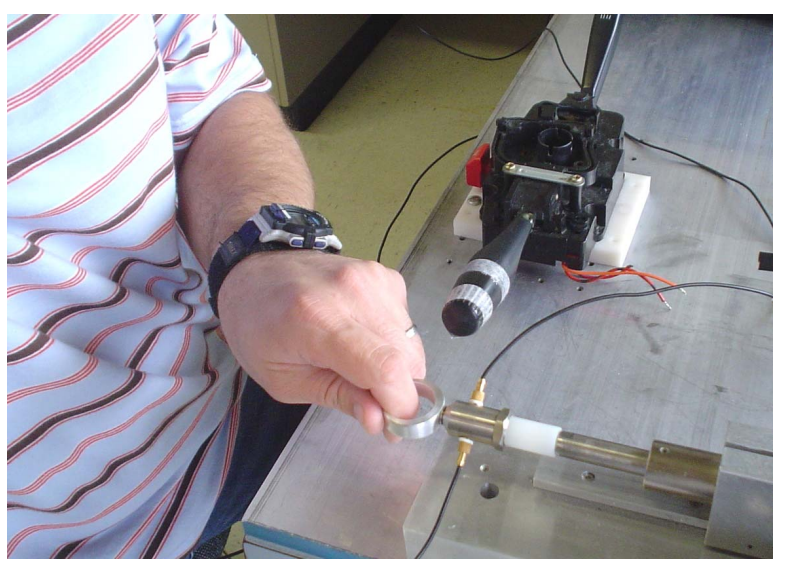

Fig. 9. Subject evaluating haptic model of turn-signal switch

of a target device. Additional experiments will be conducted to validate this assertion.

In addition to the quantitative ratings supplied by subjects in these experiments, users were also asked to provide comments regarding the model quality. Most users expressed surprise at the realism of the haptic models. Most subjects related that their strategy in rating each model involved examining the crispness and force level of the detents compared to the physical turn-signal switch. Some subjects indicated that certain model realizations felt too "active" in certain portions of the stroke, which suggests that constrained optimization methods should be explored to ensure that model parameter estimates fall within a range that guarantees model passivity. Certain subjects rated the more passive models higher than those that exhibited any vibration.

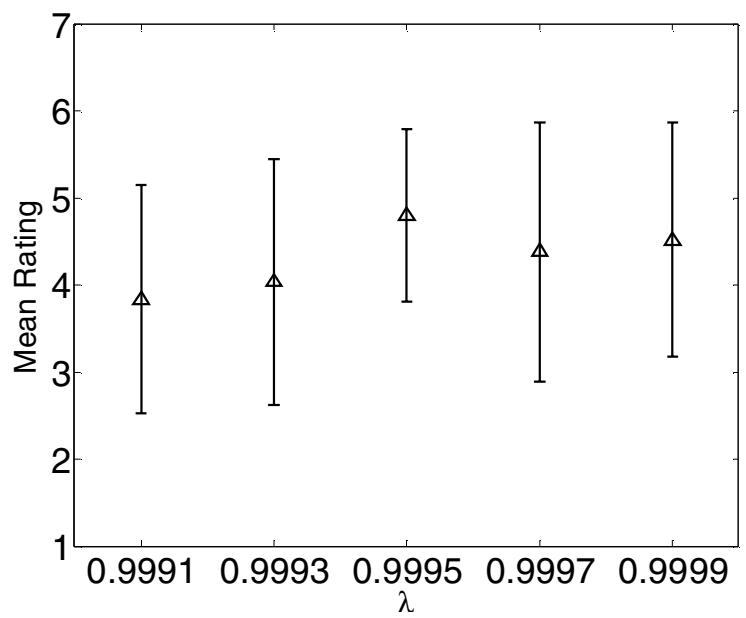

Fig. 10. Mean user ratings by forgetting factor, for $x=1 \mathrm{~mm}$ 

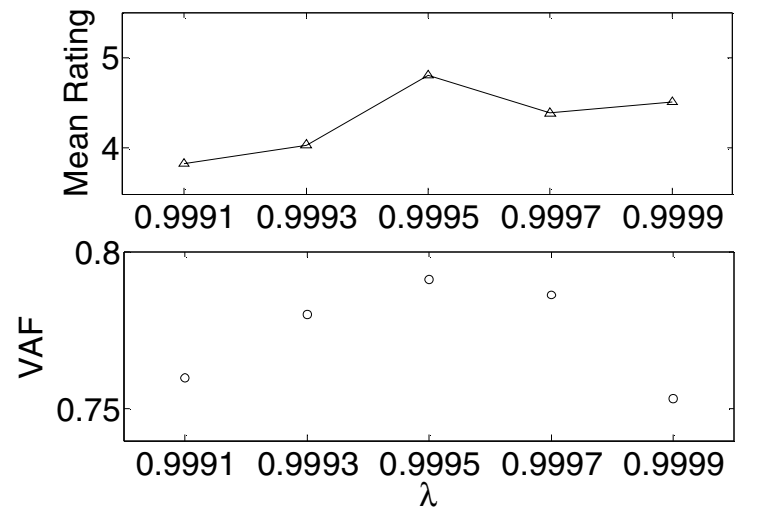

Fig. 11. Mean user ratings and VAF by forgetting factor, for $x=1 \mathrm{~mm}$

\section{Conclusion}

The method presented in this paper was shown to successfully model the feel of an automotive turnsignal switch. The method is based on a general nonlinear model structure and parameter estimation method that provide the versatility needed to model a wide class of passive mechanical devices. The effectiveness of the method was validated through traditional engineering measures (i.e., the VAF) and through subjective playback experiments. Future refinements to the method will include investigation of other parameter estimation methods and model structures, adaptation of the method to estimate models for 2-DOF devices, and use of a general robotic device to apply perturbation inputs, rather than the custombuilt instrumented probe used in the present work.

\section{Acknowledgments}

This work was supported by NSF Grant DMI 9978603

\section{References}

[1] B. Allotta, V. Colla, and G. Bioli, "A mechatronic device for simulating push-buttons and knobs," Proc. IEEE International Conference on Multimedia Computing and Systems, Florence, Italy, 1999, pp. 636642.

[2] M. Angerilli, A. Frisoli, F. Salsedo, S. Marcheschi, and M. Bergamasco, "Ha ptic simulation of an automotive manual gearshift," Proc. 10th IEEE International Workshop on Robot and Human Interactive Communication (ROMAN 2001), Paris, France, 2001, pp. 170-175.
[3] D.W. Weir, M. Peshkin, J.E. Colgate, P. Buttolo, J. Rankin, and M. Johnston, "The Haptic Profile: Capturing the feel of switches," Proc. 12th International Symposium on Haptic Interfaces for Virtual Environments and Teleoperator Systems, Chicago, IL, 2004, pp. 186-93.

[4] M. Nagurka and R. Marklin, "Measurement of stiffness and damping characteristics of computer keyboard keys," Journal of Dynamic Systems, Measurement and Control, Transactions of the ASME, vol. 127, pp. 283288, 2005.

[5] K.E. MacLean, "'Haptic Camera': A technique for characterizing and playing back haptic properties of real environments," Proc. ASME Dynamic Systems and Control Division, Atlanta, GA, 1996, pp. 459-467.

[6] M.B. Colton and J.M. Hollerbach, "Identification of nonlinear passive devices for haptic simulations," Proc. of World Haptics, Pisa, Italy, 2005, pp. 363-368.

[7] M.B. Colton and J.M. Hollerbach, "Reality-based haptic force models of buttons and switches," submitted to IEEE International Conference on Robotics and Automation (ICRA '07), Rome, Italy, 2007.

[8] G.C. Goodwin and K.S. Sin, Adaptive Filtering Prediction and Control. Englewood Cliffs, N.J.: Prentice-Hall, 1984. 